\title{
Tackling chronic migraine: current perspectives
}

\author{
This article was published in the following Dove Press journal: \\ Journal of Pain Research \\ 8 April 2014 \\ Number of times this article has been viewed
}

\section{Francisco Javier Carod- Artal}

Neurology Department, Raigmore Hospital, Inverness, UK; Universitat Internacional de Catalunya, Barcelona, Spain

\section{Video abstract}

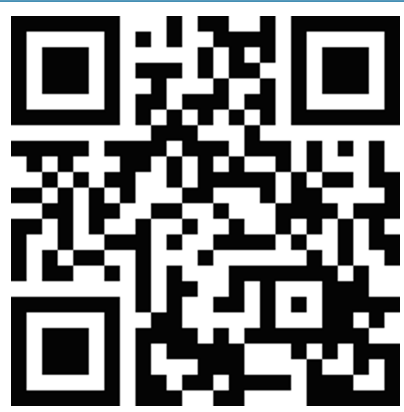

Point your SmartPhone at the code above. If you have a QR code reader the video abstract will appear. Or use: http://dvpr.es/lgoj66V

Correspondence: Francisco Javier CarodArtal

Neurology Department, Raigmore

Hospital, Old Perth Road,

IV2 3UJ, Inverness, UK

Tel +44 I4 63706229

Email javier.carodartal@nhs.net
Abstract: In the last decade, several diagnostic criteria and definitions have been proposed for chronic migraine (CM). The third edition of the International Classification of Headache Disorders -3 beta, published in 2013, has revised CM diagnostic criteria. CM is defined as "headache occurring on 15 or more days per month for more than 3 months, which has the features of migraine headache on at least 8 days per month." Patients who meet the criteria for CM and for medication-overuse headache should be given both diagnoses. Worldwide, CM prevalence ranges $1 \%-3 \%$, and its incidence has been estimated to be $2.5 \%$ per year. CM is associated with disability and poor quality of life. Modifiable risk factors include (among others): migraine progression (defined as an increase in frequency and severity of migraine attacks); medication and caffeine overuse; obesity; stressful life events; and snoring. CM patients have a significantly higher frequency of some comorbid conditions, including chronic pain, psychiatric disorders, respiratory illness, and some vascular risk factors. Management includes identification and control of comorbidities and risk factors that predispose to $\mathrm{CM}$; treatment and prevention for medication overuse; early treatment for migraine attacks; and an adequate preventive therapy for CM. Several randomized controlled clinical trials have shown the efficacy of topiramate, amitriptyline, onabotulinumtoxinA, and cognitive-behavioral therapy in CM.

Keywords: chronic daily headache, chronic migraine, epidemiology, medication overuse headache, risk factors, treatment

\section{Introduction}

Chronic daily headache comprises a group of heterogeneous primary headache disorders with a minimum duration of 4 hours per day and have occurred 15 or more days per month during the last 3 months. The third edition of the International Classification of Headache Disorders (ICHD)-3 beta, published in 2013, has comprehensively defined several primary chronic headache disorders, such as chronic migraine (CM), chronic tension-type headache, new daily persistent headache, and chronic hemicrania continua (Table 1). ${ }^{1}$ In this article, the updated diagnostic criteria, risk factors, and treatment for $\mathrm{CM}$ will be reviewed.

From the historical point of view, the concept, classification, and nomenclature of CM have evolved in the literature. CM was first defined in the second edition of the ICHD in 2004, as a complication of migraine. ${ }^{2}$ Diagnostic criteria required the presence of migraine without aura on 15 or more days per month during the last 3 months in the absence of medication overuse. This definition was judged to be very restrictive. For this reason, it was updated in the 2006 revised criteria. ${ }^{3,4}$ The ICHD-II revised criteria for $\mathrm{CM}$ required the patient to have: headache on more than 15 days 
Table I Classification of chronic primary headaches

\begin{tabular}{ll}
\hline Duration & Frequency $\geq \mathbf{I 5}$ days/month \\
\hline Long ( $\geq 4$ hours) & Chronic migraine \\
& Chronic tension-type headache \\
& Hemicrania continua \\
& New daily persistent headache \\
Short ( $<4$ hours) & Chronic cluster headache \\
& Chronic paroxysmal hemicrania \\
& SUNCT
\end{tabular}

Abbreviation: SUNCT, short-lasting, unilateral, neuralgiform headache attacks with conjunctival injection and tearing.

per month for longer than 3 months; migraine on at least eight of these monthly headache days; and no medication overuse. The updated ICHD-3 beta diagnostic criteria are summarized in Table 2. Now, overuse medication is not considered exclusion criteria for $\mathrm{CM}$.

Some authors think that the definition of CM is still unsatisfactory. ${ }^{5}$ The term "transformed migraine" was proposed to indicate a type of migraine that clinically has changed, worsened, or become more complicated over time. ${ }^{6}$ These authors proposed to distinguish between CM (merely an increase in the number of headache days that maintains most clinical characteristics typical of migraine) from transformed migraine. Transformed migraine would indicate a more severe form; the headache would not present the typical clinical symptoms of migraine, and it would be more difficult to treat than $\mathrm{CM}^{5}$

Although $\mathrm{CM}$ is the most common disorder seen in tertiary headache care, the knowledge about this chronic entity may be low, and many practitioners may not be aware of this. ${ }^{7}$ The proportion of $\mathrm{CM}$ patients that are diagnosed with the disorder may be as low as $25 \%$, and many of them may get a previous diagnosis of chronic sinusopathy, cervicogenic headache, or depression - among others. ${ }^{8}$

\section{Epidemiology}

\section{Prevalence and incidence studies}

Migraine is a chronic disorder with episodic relapses as other conditions such as angina and epilepsy. Worldwide, prevalence of migraine has been estimated at $14.7 \%$. Migraine was ranked as the third most common disease in the Global Burden of Disease Survey 2010 by the Institute for Health Metrics and Evaluation (Seattle, WA, USA), ranking seventh among specific cause of disability. ${ }^{9}$

In the last few years, several epidemiological studies have analyzed the prevalence and incidence of CM. Data has suffered the bias from the application of different diagnostic criteria and definitions across studies. In addition,
Table 2 Diagnostic criteria for chronic migraine according to the ICHD-3 beta

Headache occurring on 15 or more days per month for $>3$ months, which has the features of migraine headache on at least 8 days per month Criteria A: headache (tension-type-like and/or migraine-like) on $\geq 15$ days per month for $>3$ months and fulfilling criteria $B$ and $C$ Criteria $B$ : occurring in a patient who has had at least five attacks fulfilling criteria B-D for I.I migraines without aura and/or criteria $B$ and $C$ for 1.2 migraine with aura

Criteria C: on $\geq 8$ days per month for $>3$ months, fulfilling any of the following three

- Criteria $C$ and D for I.I migraine without aura

- Criteria B and C for 1.2 migraine with aura

- Believed by the patient to be migraine at onset and relieved by a triptan or ergot derivative

Criteria D: not better accounted for by another ICHD-3 diagnosis

Criteria B-D for migraine without aura

Criteria B: headache attacks lasting 4-72 hours (untreated or unsuccessfully treated)

Criteria C: headache has at least two of the following four

characteristics

- Unilateral location

- Pulsating quality

- Moderate or severe pain intensity

- Aggravation by or causing avoidance of routine physical activity (eg, walking or climbing stairs)

Criteria $\mathrm{D}$ : during headache at least one of the following

- Nausea and/or vomiting

- Photophobia and phonophobia

Criteria $B$ and $C$ for 1.2 migraine with aura

Criteria $B$ : one or more of the following fully reversible aura symptoms

- Visual

- Sensory

- Speech and/or language

- Motor

- Brainstem

- Retinal

Criteria C: at least two of the following four characteristics

- At least one aura symptom spreads gradually over $\geq 5$ minutes, and/or two or more symptoms occur in succession

- Each individual aura symptom lasts 5-60 minutes

- At least one aura symptom is unilateral

- The aura is accompanied, or followed within 60 minutes, by headache

Abbreviation: ICHD, International Classification of Headache Disorders.

heterogeneous interpretation of medication overuse headache (MOH) occurred in some studies. Worldwide, the prevalence of chronic daily headache was estimated to range from $3 \%-5 \%$, and $\mathrm{CM}$ prevalence from $1 \%-3 \% .^{10}$

The population-based German Headache Consortium Study evaluated approximately 10,000 people aged 18-65 years and reported prevalence for chronic headache, $\mathrm{CM}$, and chronic tension-headache was $2.6 \%, 1.1 \%$, and $0.5 \%$, respectively. ${ }^{11}$ The American Migraine Prevalence and Prevention Study (AMPP), a US national longitudinal survey of severe headache, found a CM prevalence of $1 \%{ }^{12}$ 
When less-restrictive diagnostic criteria were used and patients with $\mathrm{MOH}$ were included, then the estimated global prevalence of CM ranged between $1 \%-5 \% .{ }^{13}$ In the United States, CM prevalence has been estimated at $2.5 \%$; about 3.2 million people may suffer this chronic condition. ${ }^{8}$

Incidence studies are scarce. Episodic migraine (EM) can be considered a progressive disease that may transform to $\mathrm{CM}$ at a rate of $2.5 \%-3 \%$ per year. ${ }^{14}$ The AMPP study showed that about $2.5 \%$ of patients affected by EM progressed yearly to $\mathrm{CM} ;{ }^{15}$ whereas, the EM peak usually occurs around the age of 30-35. The highest peak of CM usually happens 10 years later. CM may affect females in approximately $80 \%$ of cases. Nevertheless, CM may also affect adolescents in approximately $0.8 \%-1.8 \%$ of cases. ${ }^{16}$

\section{Disability}

$\mathrm{CM}$ has been associated with adverse outcomes including greater headache-related disability, deterioration in healthrelated quality of life, and increased direct and indirect costs when compared to EM. ${ }^{17,18}$ The AMPP study showed that CM patients significantly had higher (worse) scores in the Migraine Disability Assessment Scale when compared to EM patients (37.9\% versus $9.5 \%$, respectively). CM-related disability was also highest among females compared with males. ${ }^{12}$

In a systematic review of 34 studies, chronic daily headache and $\mathrm{CM}$ were consistently associated with greater disability and productivity loss, more consultations, more or longer hospitalizations, and higher direct costs than episodic headache. ${ }^{18}$ The loss of productive time is more costly and increases more rapidly for people with $\mathrm{CM}$ than for those with EM, as age increases. ${ }^{19}$

\section{Remission}

Studies about the prognosis and natural history of $\mathrm{CM}$ are scarce. In a study of nearly $400 \mathrm{CM}$ patients, only $26 \%$ had $\mathrm{CM}$ remission at 2 years. Remission was defined as fewer than 10 days of headache per month, and reported remission predictors were the absence of allodynia and having a lower baseline headache frequency. ${ }^{20}$

\section{Pathophysiology of chronic migraine}

Although the source of pain persistence in CM is unknown, it is thought that pain pathways may become sensitized by repeated episodes of trigeminal activation. The chronification of migraine may be a gradual process. An increased frequency of migraine attacks and a genetic susceptibility, linked to risk factors and comorbidities, may favor chronification. In this context, trigeminovascular complex central hypersensitivity could increase excitability and/or reduce pain inhibitory mechanisms. ${ }^{14}$

Trigeminovascular system can be activated during migraine attacks, either changing the modulating nociceptive inputs from the locus coeruleus and raphe nucleus in the brainstem or due to a cortical spreading depression phenomenon. ${ }^{21}$ Structural, physiologic, and biochemical alterations can occur in the brain of EM patients. Morphological changes include reduced cortical gray matter in the pain-processing areas of the brain and iron accumulation in the periaqueductal gray matter, red nucleus, and basal ganglia. The brain of $\mathrm{CM}$ patients also exhibits metabolic changes and hyperexcitability of the central nervous system and central sensitization. These changes may be more marked compared to EM. ${ }^{22}$

Increased frequency of migraine attacks is associated with changes in the key brainstem areas, basal ganglia, and cortical areas involved in pain perception. ${ }^{23} \mathrm{~A}$ high baseline number of migraine days is associated with $\mathrm{CM}$, and the risk of chronification has been associated with a higher frequency of migraine attacks. Patients who suffer 5-9 days of migraine may have a six-fold more likely chance to develop CM compared to patients with $<4$ days of headache per month. ${ }^{24}$

Cutaneous allodynia is a marker of central sensitization and an independent predictor for migraine chronification. ${ }^{25}$ Cutaneous allodynia is more often seen in CM and is correlated with the duration of migraine illness and the frequency of attacks. Peripheral sensitization (first-order sensitization) occurs during migraine attacks; trigeminal nerves and blood vessels are sensitized and result in throbbing pain that is aggravated by physical activities. Second-order neuron sensitization occurs when sensitization spreads to trigeminovascular neurons in the spinal trigeminal nucleus and cause scalp hypersensitivity or cutaneous allodynia. Finally, third-order neuron sensitization occurs when sensitization spreads to the thalamus, provoking allodynia of the trunk and limbs. ${ }^{22}$

\section{Diagnosis}

Clinical picture may resemble a combination of migraine and tension-type headache with superimposed intermittent episodes of severe migraine. The pain in CM is usually mild to moderate. Phonophobia, photophobia, nausea, and vomiting occur much less frequently as compared to EM. However, the characteristics of the headache may change not only from day to day but even within the same day. Headache attacks with or without aura as well as tension-type headaches may occur. 
Diagnosis relies on the ICHD-3 beta criteria, as no biomarkers are available for $\mathrm{CM}^{1}{ }^{1}$ Interictal calcitonin gene-related peptide (CGRP) levels in peripheral blood may be a potential biomarker for permanent trigeminovascular activation in CM. In a recent study, CGRP levels were measured in $103 \mathrm{CM}$ women, 31 matched healthy women, and 43 matched women affected by EM. Interictal CGRP levels were significantly increased in CM patients (74.9 $\mathrm{pg} / \mathrm{mL})$ compared with healthy women $(33.7 \mathrm{pg} / \mathrm{mL})$ and EM patients $(46.4 \mathrm{pg} / \mathrm{mL}){ }^{26}$

\section{Risk factors and comorbidity Risk factors}

Epidemiological studies have identified several risk factors associated with $\mathrm{CM}$; these are summarized in Table 3. Modifiable risk factors include: migraine progression (defined as an increase in frequency and severity of migraine attacks); medication overuse; obesity; depression; stressful life events; and snoring among others. ${ }^{12,27}$ Lifetime depression may be an independent risk factor for allodynia. ${ }^{25}$ The ICHD-3 suggests that the most common cause of symptoms suggestive of CM is medication overuse. Right-to-left shunt prevalence in CM may be similar to that found in EM, so patent foramen ovale is unlikely to have any significant role in $\mathrm{CM}^{28}$

Obesity defined as body mass index (BMI) $>30 \mathrm{~kg} / \mathrm{m}^{2}$ has been associated with an increased risk of $\mathrm{CM}^{29}$ Obesity has also been linked to sleep apnea, snoring, and insulin resistance status in $\mathrm{CM} .{ }^{29,30} \mathrm{BMI}$ may have a strong influence on the CM prevalence. Overweight (BMI, 25-29.9), obesity (BMI, 30-34.9), and morbid obesity (BMI, >35) have been associated with an increased risk of $\mathrm{CM}$. The odds ratio is $1.4,1.7$, and 2.2 , respectively. ${ }^{30}$

Table 3 Risk factors for chronic migraine

\begin{tabular}{l} 
Sociodemographic factors \\
Female sex \\
Older age \\
Low educational level \\
Low income \\
Genetic factors \\
Caucasian ethnicity \\
Modifiable risk factors \\
High frequency of attacks (episodic migraine) \\
Anxiety and depression \\
Stressful life events \\
Obesity \\
Heavy caffeine consumption \\
Tobacco use \\
Overuse of abortive headache medication \\
\hline
\end{tabular}

The proportion of $\mathrm{CM}$ patients that uses preventive medication is low. Only approximately one-third of CM patients may be taking adequate preventive medication. The absence of adequate preventive medication can favor the increase of migraine attacks. Population and cross-cultural studies have shown significant differences in the rate of migraine prevention. ${ }^{31}$ The second International Burden of Migraine Study (IBMS-II) showed that $<$ one-half of CM patients were currently using any preventive medication. ${ }^{32}$ The use of prior preventive medication was reported in $66 \%$, and the mean number of prophylactic medications ever used was 3.9.

\section{Medication overuse}

Many patients with CM also report some degree of medication overuse, but it is difficult to determine whether the chronic use of painkillers is the cause or the consequence of CM. Overuse has been defined as 10 days or more of intake of triptans, ergot alkaloids, mixed analgesics or opioids, and 15 days or more of analgesics/nonsteroid anti-inflammatory drugs, or the combined use of more than one substance. ${ }^{1}$

Combination of analgesics - such as butalbital/ acetaminophen/caffeine - has been frequently associated with $\mathrm{MOH}$; whereas, nonsteroidal anti-inflammatory drugs have less potential to cause MOH. Triptans may also have the potential to cause $\mathrm{MOH}$. Opioids and barbiturate combination overuse and high caffeine consumption are also risk factors for CM transformation. The AMPP study showed that chronification was most likely to occur with 5 days of butalbital use per month, 8 days of opioid use per month, 10 days of triptan or combination analgesic per month, and 10-15 days of nonsteroidal anti-inflammatory use per month. ${ }^{33}$

According to ICHD-3 beta, approximately $50 \%$ of patients diagnosed with CM may revert to EM subtype after drug withdrawal. Many headache specialists think that these patients are in a sense wrongly diagnosed as $\mathrm{CM}$. In addition, many patients who overuse medication do not improve after drug withdrawal, and the diagnosis of $\mathrm{MOH}$ would be inappropriate. For these reasons, the ICHD-3 beta recommends that for patients who meet the criteria for 1.3 chronic migraine and for 8.2 medication-overuse headache that both diagnoses should be given. ${ }^{1}$

\section{Comorbidity}

Migraine has been associated with some comorbid conditions. Chronic pain at other locations, mood disorders, some respiratory conditions, and vascular risk factors have all been 
reported in a significantly higher frequency in CM compared to EM. In the AMPP study, CM patients were approximately twice as likely to have depression, anxiety, and chronic pain. Respiratory disorders including asthma, bronchitis and chronic obstructive pulmonary disease, and cardiac risk factors, such as hypertension, diabetes, high cholesterol, and obesity, were reported to be significantly more common in CM. The odds ratio ranged from 1.43 (cardiopathy) to 1.99 (bronchitis) (Table 4). ${ }^{27}$

\section{Management of chronic migraine}

$\mathrm{CM}$ management is a complex issue that should include modification of risk factors, treatment of associated comorbidities, identification of medication overuse, treatment of acute migraine attacks, and preventive therapy. ${ }^{7}$ Adequate preventive medication use for those patients suffering EM should be started when progression occurs to prevent CM.

Early diagnosis of CM and the detection and identification of risk factors for chronification are essential. Training of primary care physicians and neurologists is needed as diagnostic criteria for CM have not yet been unanimously accepted in the past. Once CM is identified, physicians should be aware of the difficulties of treating CM. If medication overuse is identified, the withdrawal of analgesics, opioids, and caffeine should be advised.

Optimizing mood, reducing stress, practicing good sleep hygiene, and avoiding triggers are recommended to treat CM. Strategies should be started to treat risk factors and comorbid conditions including obesity, depression, high blood pressure, neck chronic pain, and sleep and mood disorders. Keeping a headache diary or calendar would give us clinical information regarding triggers, patterns of $\mathrm{MOH}$, and the frequency of headache attacks. ${ }^{34}$ The role of migraine

Table 4 Comorbidities associated with chronic migraine

\begin{tabular}{lll}
\hline & CM, \% & EM, \% \\
\hline Arthritis & 33.6 & 22.2 \\
Chronic pain & 31.5 & 15.1 \\
Anxiety & 30.2 & 18.8 \\
Depression & 30.2 & 17.2 \\
Obesity & 25.5 & 21.0 \\
Heart disease & 9.6 & 6.3 \\
HTA & 33.7 & 27.8 \\
Asthma & 24.4 & 17.2 \\
Bronchitis & 9.2 & 4.5 \\
COPD & 4.9 & 2.6 \\
Sinusitis & 45.2 & 37 \\
\hline
\end{tabular}

Note: Data from Buse et al. ${ }^{27}$

Abbreviations: CM, chronic migraine; EM, episodic migraine; HTA, hypertension; COPD, chronic obstructive pulmonary disease. nurse specialists and psychologists should be integrated into preventive programs.

\section{Preventive medication}

Preventive medication for CM includes antiepileptic drugs (topiramate, sodium valproate), antidepressants (amitriptyline), and onabotulinumtoxinA (155-195 units). Treatment should be started with topiramate, perhaps the drug with the highest evidence to treat CM. OnabotulinumtoxinA could be considered when at least two consecutive trials of other preventive drugs have failed. Compliance needs to be assessed, as many CM patients may fail to take preventive medication. Observational studies and pooled data from randomized trials have demonstrated poor adherence to migraine prophylaxis. ${ }^{35}$

\section{Antiepileptic/antidepressants drugs}

Several double-blind, randomized, placebo-controlled clinical trials showed that topiramate may cause a significant reduction of migraine days in $\mathrm{CM}$ patients. In the European Topiramate in Chronic Migraine (TOPCHROME) study, 32 patients received topiramate (mean dose, $100 \mathrm{mg}$ / day), and 27 patients received a placebo. Topiramate significantly reduced the mean number of monthly migraine days by 3.5 compared with the placebo, and $22 \%$ of $\mathrm{CM}$ patients had a $50 \%$ or greater reduction in the mean number of headache days per month. ${ }^{36}$ In the US, a randomized clinical trial that included $306 \mathrm{CM}$ patients evaluated the efficacy of topiramate in CM. Patients received a dose of $100 \mathrm{mg}$ per day, and a decrease in mean monthly headache days was observed (5.8 days for topiramate versus 4.7 for placebo treatment). ${ }^{37}$ Both studies demonstrated the efficacy and safety of topiramate as preventive medication for CM patients with and without medication overuse. The post hoc analysis of CM patients with overuse medication concluded that detoxification prior to initiating prophylactic therapy may not be required if medication overuse is present. ${ }^{38}$

The efficacy of sodium valproate was evaluated in a small group of $29 \mathrm{CM}$ patients and 41 chronic tension-type headache patients. Patients were randomized to $500 \mathrm{mg}$ twice a day or placebo for 3 months, and a significant improvement in the severity and frequency of pain was reported in the $\mathrm{CM}$ subgroup. ${ }^{39}$ Other randomized clinical trials have assessed the efficacy of tizanidine (titrated to target dose of $24 \mathrm{mg}$ ) and gabapentin (titrated to 2,400 $\mathrm{mg}$ daily) in $\mathrm{CM}$ and a significantly lower rate of headache days as compared to placebo were found. ${ }^{40,41}$ 
The Amitriptyline Versus Placebo Study Group published a reanalysis of a clinical trial that included 391 subjects. For the subgroup with chronic daily headache, amitriptyline titrated to $100 \mathrm{mg}$ daily was significantly superior to placebo at 8 weeks and at 16 weeks. In addition, there was a trend for amitriptyline to be superior to placebo at 12 weeks and at 20 weeks, but this did not reach significance. ${ }^{42}$

\section{OnabotulinumtoxinA}

Botulinum toxin is a neurotoxin produced by anaerobic bacterium Clostridium botulinum that can improve several painful conditions including migraine. Although its antinociceptive mechanisms have not been clearly understood, onabotulinumtoxinA inhibits the release of nociceptive mediators such as substance P, CGRP, and glutamate from the peripheral terminals of primary afferents. The two Phase III REsearch Evaluating Migraine Prophylaxis Therapy (PREEMPT) clinical trials showed that onabotulinumtoxinA is a safe, well-tolerated, and effective prophylactic therapy for CM patients..$^{4-45}$

The protocol of the PREEMPT study required the injection of 150 units of onabotulinumtoxinA in 31 fixed sites at a fixed-dose of five units in each muscle. The injected muscles included procerus, bilateral corrugators, frontalis, temporalis, occipitalis, cervical, paraspinal, and superior trapezius muscles. ${ }^{46}$

A total of 1,384 patients were randomized to onabotulinumtoxinA $(\mathrm{n}=688)$ or placebo $(\mathrm{n}=696)$ in the double-blind phase of PREEMPT trials. At 24 weeks, around $47 \%$ of onabotulinumtoxinA-treated patients had a decrease in the frequency of headache days higher than $50 \%$ compared with $35 \%$ of placebo-treated patients. A pooled analysis showed that onabotulinumtoxinA treatment significantly reduced headache-day frequency versus placebo in patients with $\mathrm{CM}$ at week 56. A significant reduction in the frequency of migraine days and cumulative headache hours on headache days was also observed. ${ }^{47}$

The $62.4 \%$ of onabotulinumtoxin A-treated patients and the $51.7 \%$ of placebo-treated patients reported an adverse event. Most adverse events were mild to moderate in severity, and only $3.8 \%$ of onabotulinumtoxinA-treated patients (and $1.2 \%$ of placebo-treated patients) discontinued therapy due to adverse events. ${ }^{48}$ OnabotulinumtoxinA is an expensive preventive treatment for $\mathrm{CM}$. However, cost could be reduced by less triptan use for the acute migraine attacks. ${ }^{49} \mathrm{~A}$ costeffectiveness analysis showed that onabotulinumtoxinA reduced headache days by an estimated 38 days per year at a cost of $£ 18$ per headache avoided in adults with $\mathrm{CM}$ in United Kingdom. ${ }^{50}$
A few studies have compared topiramate and onabotulinumtoxin A in CM prevention and found similar efficacy. In a study of $60 \mathrm{CM}$ patients who were randomized to 200 units of onabotulinumtoxinA versus topiramate $200 \mathrm{mg}$ daily, a reduction $>50 \%$ in the mean number of headache days were observed in $41 \%$ of onabotulinumtoxinA-treated patients and $43 \%$ of topiramate-treated ones. ${ }^{51}$ The second study recruited a small sample of 59 subjects that were randomized to topiramate (200 mg daily) or onabotulinumtoxinA (200 units). At week 12, a decrease in the mean number of headache days per month was similar in both groups (12.4 versus 13.8 days). ${ }^{52}$

\section{Open studies and combination therapies in CM}

The results of several open-label trials have been published and they suggest some degree of efficacy for zonisamide (400 mg daily), ${ }^{53,54}$ pregabalin (150 mg twice-a-day), ${ }^{55}$ flunarizine (10 mg daily), ${ }^{56}$ atenolol (50 mg daily), ${ }^{57}$ and memantine (10-20 mg daily), ${ }^{58}$ to treat CM. However, more evidence and further randomized clinical trials are needed to demonstrate their efficacy in CM.

There are only a few studies that have evaluated the efficacy of combination therapies in CM. A randomized, placebo-controlled trial of propranolol added to topiramate in CM has been published. ${ }^{59}$ The result of this study showed that the addition of propranolol long-acting (240 mg daily) to topiramate $50-100 \mathrm{mg}$ per day does not add benefit when $\mathrm{CM}$ is inadequately controlled with topiramate alone. In another small pilot trial of 28 subjects, the combination of sumatriptan $85 \mathrm{mg}$ plus naproxen $500 \mathrm{mg}$ in a combination tablet did not reduce headache frequency over a 3-month period. ${ }^{60}$ Nevertheless, further clinical trials are needed to search for better combination therapies for CM patients.

An unresolved question is whether migraine patients with medication overuse should taper off the overused medication before starting any preventive medication. The post hoc analysis of the European and US topiramate clinical trials and the results of onabotulinumtoxinA trials suggest some type of benefit for patients overusing triptans and other medications..$^{38,45}$

\section{Cognitive-behavioral therapy and other therapies}

Several studies have assessed the role of complementary and alternative therapies in CM. Biobehavioral techniques used in chronic headache include relaxation training, thermal biofeedback, electromyography biofeedback, and cognitivebehavioral therapy. 
Although biofeedback, relaxation technique, and cognitive-behavioral therapies have demonstrated efficacy in the prevention of $\mathrm{EM}$, data regarding efficacy in $\mathrm{CM}$ are limited. ${ }^{8}$ Behavioral sleep modification proved to be effective for transformed migraine. ${ }^{61}$

Relaxation training and biofeedback are directed toward the sensory component that involves the perception of physical sensations including pain. Biofeedback training targets those physical responses that are believed to contribute to increase the susceptibility and maintenance of chronic headache. Cognitive and behavioral strategies focus on the reactive component of the pain experience. ${ }^{62}$

The rationale of cognitive-behavioral therapies in $\mathrm{CM}$ is that chronic headache should be recognized and thoroughly examined by the patient to be successfully managed. Being aware of factors that precipitate or aggravate CM should allow patients to progressively modulate the frequency and duration of their attacks. ${ }^{63}$ Cognitive-behavioral therapy may also be indicated to assess compliance to preventive medication and to assess modifiable risk factors for CM. ${ }^{62}$ Other reasons that cause CM patients to seek cognitivebehavioral therapy and other nonpharmacological treatments include poor tolerance and low response to preventive medication, history of overuse of medication, pregnancy, stress, and deficient stress/pain coping strategies. ${ }^{62}$

The goals of cognitive-behavioral therapy in CM are summarized in Table 5. CM patients are taught self-regulation techniques and cognitive and behavioral coping skills. Diaries and headache calendars may be helpful to adequately register the patient's treatment progress. CM patients are also encouraged to modify risk factors to reduce frequency and severity of headache.

Cognitive-behavioral therapy can be helpful as part of a combined treatment, and it should be integrated with pharmacological interventions, physical, and aerobic training. A randomized clinical trial evaluated the efficacy of cognitive-behavioral therapy plus amitriptyline in $\mathrm{CM}$ children and adolescents aged $10-17$ years. ${ }^{64}$ Also, 135 youth

Table 5 Goals of cognitive-behavioral therapy in chronic migraine

To reduce frequency and severity of migraine attacks/days of headache

To decrease disability and improve patient's health-related quality of life

To reduce medication overuse

To diminish intake of poorly tolerated or unwanted preventive medication

To develop coping strategies for chronic headache

To enhance individual control of pain

To reduce chronic migraine-related stress

To treat modifiable risk factors for chronic migraine

Note: Data from Mathew et al. ${ }^{6}$ patients (79\% female) diagnosed with CM were recruited and randomized to the cognitive-behavioral therapy plus amitriptyline group ( $\mathrm{n}=64$ ) or the headache education plus amitriptyline group $(n=71)$. A greater reduction in headache days and migraine-related disability was observed in the cognitive-behavioral therapy plus amitriptyline group, as compared to headache education/amitriptyline group. ${ }^{64}$

Acupuncture may be beneficial when added to medical treatment. A randomized clinical trial of 66 patients evaluated the efficacy of acupuncture administered in 24 sessions over 12 weeks versus topiramate $(100 \mathrm{mg}$ daily) in CM. Although this was a nonblind study, researchers found a significant decrease in the mean number of headache days in the acupuncture branch as compared to the topiramate. ${ }^{65}$

\section{Occipital nerve stimulation}

Occipital nerve stimulation has been assessed in patients with refractory $\mathrm{CM} .{ }^{66}$ Three randomized sham-controlled trials have evaluated the efficacy of occipital nerve stimulation in $\mathrm{CM}$ at 12 weeks, and the overall results have been considered disappointing. ${ }^{66}$ In the Precision Implantable Stimulator for Migraine (PRISM) trial, 125 refractory CM patients were treated with occipital nerve stimulation or sham, and no significant improvement was observed. ${ }^{67}$

The Occipital Nerve Stimulation for the Treatment of Intractable Migraine (ONSTIM) study assessed the efficacy of occipital nerve stimulation in $75 \mathrm{CM}$ patients. After 3 months, a 50\% reduction in headache was reported in 39\% of patients who had adjustable stimulation and in 5\% who had preset stimulation.$^{68}$ However, lead migration occurred in $24 \%$ of subjects.

A recent large-scale randomized controlled multicenter study of CM patients showed that there was not a significant difference in the percentage of responders in the active compared with the control group at 12 weeks. Active group patients $(\mathrm{n}=105)$ were implanted with a neurostimulation device near the occipital nerve; whereas, 52 patients received sham stimulation. Compared with the sham-treated group, significant differences in the reduction of number of headache days, migraine-related disability, and direct reports of pain were observed in the active group. ${ }^{69}$

Another study of 30 patients reported that headache intensity and frequency were significantly lower in the "on" arm as compared to the "off" one. ${ }^{70}$ Dual occipital and supraorbital nerve stimulation have also been evaluated in a small study of 14 patients, and approximately $70 \%$ of them had a $50 \%$ or greater decrease in pain severity. ${ }^{71}$ 
Occipital nerve stimulation is a relatively safe technique although invasive and expensive, and this factor may also limit its use in clinical practice. Adverse effects have been reported and include lead migration, local acute or delayed infections, and battery depletion due to the high stimulation intensity needed in some patients. Occipital nerve stimulation can also induce paresthesias covering the great occipital nerve territory.

The position statement from the European Headache Federation concludes that the application of neurostimulation for $\mathrm{CM}$ should be considered only after all alternative drugs and behavioral therapies, as recommended by international guidelines, have failed, and medication overuse headache excluded ${ }^{66}$ The use of occipital nerve stimulation seems acceptable in CM, although it is based on limited evidence.

The effect of infiltrations on the occipital nerve in CM is unknown. An open-label study assessed the efficacy and safety of the occipital nerve block in 150 patients affected with $\mathrm{CM}$ and associated cervicogenic triggers. Thirty-seven CM patients were treated with unilateral occipital nerve block; 113 received bilateral occipital nerve blocks. Also, one-half of the patients had a $50 \%$ reduction in headache days over the month following the occipital nerve block. However, $5 \%$ of patients reported adverse events within the ensuing 72 hours. $^{72}$

\section{Conclusion}

Diagnosis, treatment, and prevention for $\mathrm{CM}$ are challenging. Educational programs should be proposed for EM patients as they should be warned about the potential risk of chronification of the disease. Early detection and management of modifiable risk factors, such as migraine progression, medication overuse, obesity, depression, and stressful life events, are also important to avoid the chronification of migraine. ${ }^{73}$

Preventive medication may be ineffective in many patients; some frequently report side effects of medication. Many of these may also suffer from $\mathrm{MOH}$ and even a subgroup of patients may suffer "intractable" or refractory CM. ${ }^{74}$ The amount of randomized clinical trials in $\mathrm{CM}$ is limited, and the number of recruited patients has been small. Different diagnostic criteria and outcomes have been used and this fact may limit comparison and generalization of their results.

\section{Disclosure}

The author reports no conflicts of interest in this work.

\section{References}

1. Headache Classification Committee of the International Headache Society (IHS). The International Classification of Headache Disorders. 3rd ed (beta version). Cephalalgia. 2013;33(9):629-808.

2. Headache Classification Subcommittee of the International Headache Society. The International Classification of Headache Disorders. 2nd ed. Cephalalgia. 2004; 24 Suppl 1:9-160.

3. Headache Classification Committee; Olesen J, Bousser MG, Diener HC, et al. New appendix criteria open for a broader concept of chronic migraine. Cephalalgia. 2006;26(6):742-746.

4. Silberstein SD, Lipton RB, Sliwinski M. Classification of daily and near-daily headaches: field trial of revised IHS criteria. Neurology. 1996;47(4):871-875.

5. Manzoni GC, Bonavita V, Bussone G, et al; ANIRCEF (Associazione Neurologica Italiana Ricerca Cefalee). Chronic migraine classification: current knowledge and future perspectives. J Headache Pain. 2011;12(6):585-592.

6. Mathew NT, Stubits E, Nigam MP. Transformation of episodic migraine into daily headache: analysis of factors. Headache. 1982; 22(2):66-68.

7. Carod-Artal FJ, Irimia P, Ezpeleta D. [Chronic migraine: definition, epidemiology, risk factors and treatment]. Rev Neurol. 2012;54(10): 629-637. Spanish [with English abstract].

8. Evans RW. A rational approach to the management of chronic migraine. Headache. 2013;53(1):168-176.

9. Vos T, Flaxman AD, Naghavi M, et al. Years lived with disability (YLD) for 1160 sequelae of 289 diseases and injuries 1990-2010: a systematic analysis for the Global Burden of Disease Study 2010. Lancet. 2012; 380(9859):2163-2196.

10. Manack AN, Buse DC, Lipton RB. Chronic migraine: epidemiology and disease burden. Curr Pain Headache Rep. 2011;15(1):70-78.

11. Schramm SH, Obermann M, Katsarava Z, Diener HC, Moebus S, Yoon MS. Epidemiological profiles of patients with chronic migraine and chronic tension-type headache. J Headache Pain. 2013; 14(1):40.

12. Buse DC, Manack AN, Fanning KM, et al. Chronic migraine prevalence, disability, and sociodemographic factors: results from the American Migraine Prevalence and Prevention Study. Headache. 2012;52(10): 1456-1470.

13. Natoli JL, Manack A, Dean B, et al. Global prevalence of chronic migraine: a systematic review. Cephalalgia. 2010;30(5):599-609.

14. Lipton RB. Tracing transformation: chronic migraine classification, progression, and epidemiology. Neurology. 2009;72(Suppl 5):S3-S7.

15. Bigal ME, Serrano D, Buse D, Scher A, Stewart WF, Lipton RB. Acute migraine medications and evolution from episodic to chronic migraine: a longitudinal population-based study. Headache. 2008;48(8): 1157-1168.

16. Lipton RB, Manack A, Ricci JA, Chee E, Turkel CC, Winner P. Prevalence and burden of chronic migraine in adolescents: results of the chronic daily headache in adolescents study (C-dAS). Headache. 2011;51(5):693-706.

17. Blumenfeld AM, Varon SF, Wilcox TK, et al. Disability, HRQoL and resource use among chronic and episodic migraineurs: results from the International Burden of Migraine Study (IBMS). Cephalalgia. 2010;31(3):301-315.

18. Lantéri-Minet M, Duru G, Mudge M, Cottrell S. Quality of life impairment, disability and economic burden associated with chronic daily headache, focusing on chronic migraine with or without medication overuse: a systematic review. Cephalalgia. 2011;31(7): $837-850$.

19. Serrano D, Manack AN, Reed ML, Buse DC, Varon SF, Lipton RB. Cost and predictors of lost productive time in chronic migraine and episodic migraine: results from the American Migraine Prevalence and Prevention (AMPP) Study. Value Health. 2013;16(1):31-38.

20. Manack A, Buse DC, Serrano D, Turkel CC, Lipton RB. Rates, predictors, and consequences of remission from chronic migraine to episodic migraine. Neurology. 2011;76(8):711-718. 
21. Moskowitz MA. The visceral organ brain: implications for the pathophysiology of vascular head pain. Neurology. 1991;41(2(Pt 1)): 182-186.

22. Mathew NT. Pathophysiology of chronic migraine and mode of action of preventive medications. Headache. 2011;51 Suppl 2:84-92.

23. Maniyar FH, Goadsby PJ. Functional imaging in chronic migraine. Curr Pain Headache Rep. 2013;17(5):333.

24. Katsarava Z, Schneeweiss S, Kurth T, et al. Incidence and predictors for chronicity of headache in patients with episodic migraine. Neurology. 2004;62(5):788-790.

25. Louter MA, Bosker JE, van Oosterhout WP, et al. Cutaneous allodynia as a predictor of migraine chronification. Brain. 2013; 136(Pt 11):3489-3496.

26. Cernuda-Morollón E, Larrosa D, Ramón C, Vega J, Martínez-Camblor P, Pascual J. Interictal increase of CGRP levels in peripheral blood as a biomarker for chronic migraine. Neurology. 2013;81(14): 1191-1196.

27. Buse DC, Manack A, Serrano D, Turkel C, Lipton RB. Sociodemographic and comorbidity profiles of chronic migraine and episodic migraine sufferers. J Neurol Neurosurg Psychiatry. 2010;81(4):428-432.

28. Guo S, Shalchian S, Gérard P, et al. Prevalence of right-to-left shunts on transcranial Doppler in chronic migraine and medication-overuse headache. Cephalalgia. 2014;34(1):37-41.

29. Fava A, Pirritano D, Consoli D, et al. Chronic migraine in women is associated with insulin resistance: a cross-sectional study. Eur J Neurol. 2014;21(2):267-272.

30. Bigal ME, Lipton RB. Obesity is a risk factor for transformed migraine but not chronic tension-type headache. Neurology. 2006;67(2): 252-257.

31. Carod-Artal FJ, Ezpeleta D, Martín-Barriga ML, Guerrero AL. Triggers, symptoms, and treatment in two populations of migraneurs in Brazil and Spain. A cross-cultural study. J Neurol Sci. 2011;304(1-2):25-28.

32. Blumenfeld AM, Bloudek LM, Becker WJ, et al. Patterns of use and reasons for discontinuation of prophylactic medications for episodic migraine and chronic migraine: results from the second international burden of migraine study (IBMS-II). Headache. 2013;53(4): 644-655.

33. Tepper SJ. Medication-overuse headache. Continuum (Minneap Minn). 2012;18(4):807-822.

34. Schulman E, McGeeney BE. Current concepts in refractory migraine. Curr Treat Options Neurol. 2013;15(1):40-55.

35. Hepp Z, Bloudek LM, Varon SF. Systematic review of migraine prophylaxis adherence and persistence. J Manag Care Pharm. 2014;20(1):22-33.

36. Diener HC, Bussone G, Van Oene JC, Lahaye M, Schwalen S, Goadsby PJ; TOPMAT-MIG-201(TOP-CHROME) Study Group. Topiramate reduces headache days in chronic migraine: A randomized, double-blind, placebo-controlled study. Cephalalgia. 2007;27(7):814-823.

37. Silberstein S, Lipton R, Dodick D, et al. Topiramate treatment of chronic migraine: a randomized, placebo-controlled trial of quality of life and other efficacy measures. Headache. 2009;49(8):1153-1162.

38. Diener HC, Dodick DW, Goadsby PJ, et al. Utility of topiramate for the treatment of patients with chronic migraine in the presence or absence of acute medication overuse. Cephalalgia. 2009;29(10): 1021-1027.

39. Yurekli VA, Akhan G, Kutluhan S, Uzar E, Koyuncuoglu HR, Gultekin F. The effect of sodium valproate on chronic daily headache and its subgroups. J Headache Pain. 2008;9(1):37-41.

40. Saper JR, Lake AE 3rd, Cantrell DT, Winner PK, White JR. Chronic daily headache prophylaxis with tizanidine: a double-blind, placebo-controlled, multicenter outcome study. Headache. 2002;42(6):470-482.

41. Spira PJ, Beran RG; Australian Gabapentin Chronic Daily Headache Group. Gabapentin in the prophylaxis of chronic daily headache: a randomized, placebo-controlled study. Neurology. 2003;61(12): 1753-1759.

42. Couch JR, Amitriptyline Versus Placebo Study Group. Amitriptyline in the prophylactic treatment of migraine and chronic daily headache. Headache. 2011;51(1):33-51.
43. Aurora SK, Dodick DW, Turkel CC, et al; PREEMPT 1 Chronic Migraine Study Group. OnabotulinumtoxinA for treatment of chronic migraine: results from the double-blind, randomized, placebocontrolled phase of the PREEMPT 1 trial. Cephalalgia. 2010;30(7): 793-803.

44. Diener HC, Dodick DW, Aurora SK, et al; PREEMPT 2 Chronic Migraine Study Group. OnabotulinumtoxinA for treatment of chronic migraine: results from the double-blind, randomized, placebo-controlled phase of the PREEMPT 2 trial. Cephalalgia. 2010; 30(7):804-814.

45. Aurora SK, Dodick DW, Diener HC, et al. OnabotulinumtoxinA for chronic migraine: efficacy, safety, and tolerability in patients who received all five treatment cycles in the PREEMPT clinical program. Acta Neurol Scand. 2014;129(1):61-70.

46. Blumenfeld A, Silberstein SD, Dodick DW, Aurora SK, Turkel CC, Binder WJ. Method of injection of onabotulinumtoxinA for chronic migraine: a safe, well-tolerated, and effective treatment paradigm based on the PREEMPT clinical program. Headache. 2010;50(9): 1406-1418.

47. Aurora SK, Winner P, Freeman MC, et al. OnabotulinumtoxinA for treatment of chronic migraine: pooled analyses of the 56-week PREEMPT clinical program. Headache. 2011;51(9):1358-1373.

48. Dodick DW, Turkel CC, DeGryse R, et al; PREEMPT Chronic Migraine Study Group. OnabotulinumtoxinA for treatment of chronic migraine: pooled results from the double-blind, randomized, placebo-controlled phases of the PREEMPT clinical program. Headache. 2010;50(6): 921-936.

49. Oterino A, Ramón C, Pascual J. Experience with onabotulinumtoxinA (BOTOX) in chronic refractory migraine: focus on severe attacks. J Headache Pain. 2011;12(2):235-238.

50. Batty AJ, Hansen RN, Bloudek LM, et al. The cost-effectiveness of onabotulinumtoxinA for the prophylaxis of headache in adults with chronic migraine in the UK. J Med Econ. 2013;16(7):877-887.

51. Mathew NT, Jaffri SF. A double-blind comparison of onabotulinumtoxina (BOTOX) and topiramate (TOPAMAX) for the prophylactic treatment of chronic migraine: a pilot study. Headache. 2009;49(10): 1466-1478.

52. Cady RK, Schreiber CP, Porter JA, Blumenfeld AM, Farmer KU. A multi-center double-blind pilot comparison of onabotulinumtoxinA and topiramate for the prophylactic treatment of chronic migraine. Headache. 2011;51(1):21-32.

53. Bermejo PE, Dorado R. Zonisamide for migraine prophylaxis in patients refractory to topiramate. Clin Neuropharmacol. 2009;32(2): 103-106.

54. Pascual-Gómez J, Gracia-Naya M, Leira R, et al. [Zonisamide in the preventive treatment of refractory migraine]. Rev Neurol. 2010;50: 129-132. Spanish [with English abstract].

55. Calandre EP, Garcia-Leiva JM, Rico-Villademoros F, Vilchez JS, Rodriguez-Lopez CM. Pregabalin in the treatment of chronic migraine: an open-label study. Clin Neuropharmacol. 2010;33(1):35-39.

56. Gracia-Naya M, Ríos C, García-Gomara MJ, et al. A comparative study of the effectiveness of topiramate and flunarizine in independent series of chronic migraine patients without medication abuse. Rev Neurol. 2013;57(8):347-353. Spanish [with English abstract].

57. Edvardsson B. Atenolol in the prophylaxis of chronic migraine: a 3-month open-label study. Springerplus. 2013;2:479.

58. Bigal M, Rapoport A, Sheftell F, Tepper D, Tepper S. Memantine in the preventive treatment of refractory migraine. Headache. 2008;48(9): 1337-1342.

59. Silberstein SD, Dodick DW, Lindblad AS, et al; Chronic Migraine Treatment Trial Research Group. Randomized, placebo-controlled trial of propranolol added to topiramate in chronic migraine. Neurology. 2012;78(13):976-984.

60. Cady R, Nett R, Dexter K, Freitag F, Beach ME, Manley HR. Treatment of chronic migraine: a 3-month comparator study of naproxen sodium vs SumaRT/Nap. Headache. 2014;54(1):80-93.

61. CalhounAH, Ford S. Behavioral sleep modification may revert transformed migraine to episodic migraine. Headache. 2007;47(8):1178-1183. 
62. Weeks RE. Application of behavioral therapies in adult and adolescent patients with chronic migraine. Neurol Sci. 2013;34 Suppl 1: S11-S17.

63. Pistoia F, Sacco S, Carolei A. Behavioral therapy for chronic migraine. Curr Pain Headache Rep. 2013;17(1):304.

64. Powers SW, Kashikar-Zuck SM, Allen JR, et al. Cognitive behavioral therapy plus amitriptyline for chronic migraine in children and adolescents: a randomized clinical trial. JAMA. 2013;310(24):2622-2630.

65. Yang CP, Chang MH, Liu PE, et al. Acupuncture versus topiramate in chronic migraine prophylaxis: a randomized clinical trial. Cephalalgia. 2011;31(15):1510-1521.

66. Martelletti P, Jensen RH, Antal A, et al. Neuromodulation of chronic headaches: position statement from the European Headache Federation. J Headache Pain. 2013;14(1):86.

67. Lipton R, Goadsby P, Cady R, et al. PRISM study: occipital nerve stimulation for treatment-refractory migraine. Cephalalgia. 2009;29:30.

68. Saper JR, Dodick DW, Silberstein SD, McCarville S, Sun M, Goadsby PJ; ONSTIM Investigators. Occipital nerve stimulation for the treatment of intractable chronic migraine headache: ONSTIM feasibility study. Cephalalgia. 2011;31(3):271-285.
69. Silberstein SD, Dodick DW, Saper J, et al. Safety and efficacy of peripheral nerve stimulation of the occipital nerves for the management of chronic migraine: Results from a randomized, multicenter, double-blinded, controlled study. Cephalalgia. 2012;32(16): 1165-1179.

70. Serra G, Marchioretta F. Occipital nerve stimulation for chronic migraine: a randomized trial. Pain Physician. 2012;15(3):245-253.

71. Hann S, Sharan A. Dual occipital and supraorbital nerve stimulation for chronic migraine: a single-center experience, review of literature, and surgical considerations. Neurosurg Focus. 2013;35:E9.

72. Weibelt S, Andress-Rothrock D, King W, Rothrock J. Suboccipital nerve blocks for suppression of chronic migraine: safety, efficacy, and predictors of outcome. Headache. 2010;50(6):1041-1044.

73. Manzoni GC, Camarda C, Torelli P. Chronification of migraine: what clinical strategies to combat it? Neurol Sci. 2013;34 Suppl 1: S57-S60.

74. Goadsby PJ, Schoenen J, Ferrari MD, Silberstein SD, Dodick D. Towards a definition of intractable headache for use in clinical practice and trials. Cephalalgia. 2006;26(9):1168-1170.
Journal of Pain Research

\section{Publish your work in this journal}

The Journal of Pain Research is an international, peer-reviewed, open access, online journal that welcomes laboratory and clinical findings in the fields of pain research and the prevention and management of pain. Original research, reviews, symposium reports, hypothesis formation and commentaries are all considered for publication.

\section{Dovepress}

The manuscript management system is completely online and includes a very quick and fair peer-review system, which is all easy to use. Visit http://www.dovepress.com/testimonials.php to read real quotes from published authors. 